\title{
Coronary artery spasm as the probable cause of cardiac arrest immediately after the induction of spinal anesthesia \\ - A case report -
}

\section{Jung A Kim ${ }^{1}$, Chan Jong Chung', Kyoung Sub Yoon', Jeong In Hong', Seung Cheol Lee', Sang Yoong Park', So Ron Choi', Dong Hyun Lee ${ }^{2}$, and Jin-Heon Jeong ${ }^{2}$}

\section{Departments of ${ }^{1}$ Anesthesiology and Pain Medicine, ${ }^{2}$ Intensive Care Medicine, Dong-A} University Hospital, Busan, Korea

\author{
Corresponding author \\ So Ron Choi, M.D., Ph.D. \\ Department of Anesthesiology and \\ Pain Medicine, Dong-A University \\ Hospital, 26 Daesingongwon-ro, Seo- \\ gu, Busan 49201, Korea \\ Tel: 82-51-240-5390 \\ Fax: 82-51-247-7819 \\ E-mail: choisr@dau.ac.kr \\ ORCID \\ http://orcid.org/0000-0002-4173-8939
}

\begin{abstract}
A 72-year-old man underwent spinal anesthesia for artificial urinary sphincter placement for urinary incontinence. After the block level was confirmed below T6, $1 \mathrm{~g}$ of cefotetan, which had not shown any reaction on skin test, was administered as a prophylactic antibiotic. The patient began complaining of chest discomfort and dyspnea shortly after injection. ST elevation appeared on the electrocardiogram and the patient's pulse could not be palpated. Accordingly, cardiopulmonary resuscitation was performed for 5 minutes; the patient recovered spontaneous circulation. The patient was diagnosed as experienced coronary artery spasm by coronary angiography with spasm test. Because coronary artery spasm can also develop in patients with no history of coronary artery disease and under spinal anesthesia, careful observation, suspicion of coronary artery spasm and prompt response to hemodynamic and electrocardiogram changes are necessary.
\end{abstract}

Key Words: Conduction anesthesia, Coronary vasospasm, Heart arrest, Spinal anesthesia.

Variant angina was first described by Prinzmetal et al. [1], and is one of the major causes of intraoperative or perioperative myocardial ischemia. If not treated immediately, it can lead to myocardial infarction and death [2]. Coronary artery spasm (CAS) rarely occurs during anesthesia, and occurrences of CAS during spinal anesthesia leading to circulatory collapse are especially rare [3]. We report a case involving a patient with no history of coronary artery disease, who experienced cardiac arrest accompanied by ST elevation immediately after induction of spinal anesthesia and administration of prophylactic antibiotic. The patient recovered spontaneous circulation after cardiopulmonary resuscitation (CPR). The patient was eventually diagnosed with CAS based on an- giography results. The only suspicious cause for CAS was hemodynamic change due to spinal anesthesia and emotional stress.

\section{CASE REPORT}

The patient described in this report provided informed consent for publication of anonymized case details.

A 72-year-old man (167 cm tall, $74.9 \mathrm{~kg}$ ) was admitted to the authors' hospital to undergo elective artificial urinary sphincter placement for incontinence. The patient was diagnosed with hypertension 20 years previously and was well controlled with carvedilol. Laboratory investigations,

This is an Open Access article distributed under the terms of the Creative Commons Attribution Non-Commercial License (http://creativecommons.org/licenses/by-nc/4.0) which permits unrestricted non-commercial use, distribution, and reproduction in any medium, provided the original work is properly cited.

Copyright (C) the Korean Society of Anesthesiologists, 2018 
electrocardiogram (ECG), chest X-rays and echocardiograms performed before surgery revealed no abnormal findings. The patient was administered $5 \mathrm{mg}$ of diazepam orally for premedication.

The patient's vital signs at the time of admission to the operating room were: blood pressure (BP) 133/62 mmHg; heart rate 56 beats/min; peripheral oxygen saturation $97 \%$; and normal ECG. Spinal anesthesia was induced by administration of heavy bupivacaine (13 $\mathrm{mg})$ and fentanyl $(20 \mu \mathrm{g})$ at L4-5; 7 minutes later, anesthesia was confirmed below T6 using the cold test. Cefotetan (1 g), which had shown negative skin test results, was administered before operation. Following administration, the patient complained of dyspnea and constant chest pain. The sensory block level was checked and was still below T6. The patient began to lose consciousness. BP decreased to $62 / 35 \mathrm{mmHg}$ and heart rate was steady at 60 beats/min. ST elevation was suddenly observed on ECG, and the patient's arterial pulse was not palpable any longer. Accordingly, CPR was performed and $50 \mathrm{mg}$ of rocuronium was administered; the patient was then intubated to assist respiration. After 5 minutes CPR, the patient's pulse could be palpated, and ECG revealed ST depression. The patient was still hypotensive (systolic BP $50 \mathrm{mmHg}$ ), despite the continuous administration of isosorbide dinitrate $(3 \mathrm{mg} / \mathrm{h})$, dopamine (20 $\mathrm{g} / \mathrm{kg} / \mathrm{min})$, and epinephrine (20 $\mu \mathrm{g} / \mathrm{min})$. Ventricular fibrillation occurred 15 minutes after CPR was discontinued, which reverted to normal sinus rhythm after defibrillation with 150 J shocks. Twelve-lead ECG revealed normal sinus rhythm and transesophageal echocardiography demonstrated no problems associated with heart motility. BP gradually began to rise until systolic pressure was stable and above 100 mmHg. The surgery was canceled and the patient, who at this point was in a stupor, was transferred to the intensive care unit (ICU).

Laboratory test showed Troponin-I was increased to $3.1673 \mathrm{ng} / \mathrm{ml}$. The patient was placed on mechanical ventilation while receiving dexmedetomidine, remifentanil and vecuronium. Therapeutic hypothermia was induced for 24 hours. Brain magnetic resonance imaging on post-operative day (POD) 3 confirmed no hypoxic brain damage. On POD 4, the patient recovered spontaneous respiration and was in a drowsy state. After undergoing conservative treatment for rib fracture resulting from $\mathrm{CPR}$, the patient was discharged on POD 17.

Anaphylactic shock induced by cefotetan and CAS were the suspected causes of cardiac arrest. While the patient showed mildly positive results in a skin test to cefotetan for the second time after ICU admission, serum tryptase (18.7 $\mathrm{ng} / \mathrm{ml}$ ) on POD 1 , and specific immunoglobulin E (cefaclor [c7]), penicillin $\mathrm{G}(\mathrm{c} 1$ ), penicillin $\mathrm{V}$ (c2), ampicillin (c5), and amoxycillin (c6) tests on POD 4 were all class 0 , and total immunoglobulin E was $644 \mathrm{IU} / \mathrm{ml}$, eliminating the possibility of antibiotic-induced anaphylaxis as the cause of cardiac arrest. Cefotetan was later administered in the ICU and the patient exhibited no allergic reaction.

One month after discharge, coronary angiography and a spasm test were performed, which revealed $60 \%$ to $70 \%$ stenosis in the mid left anterior descending coronary artery that was alleviated with nitroglycerin administration (Fig. 1). The patient was diagnosed with CAS and was prescribed nifedip-
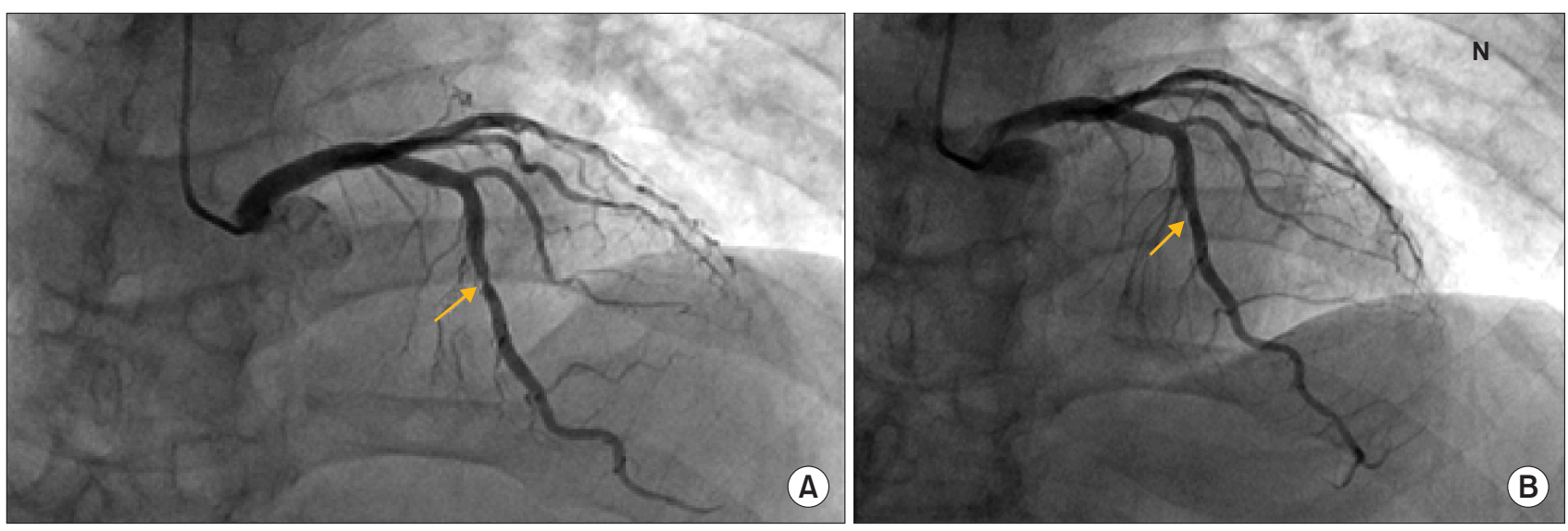

Fig. 1. (A) Coronary angiography revealing positive spasm test in the mid left anterior descending coronary artery (arrow). (B) Response of the stenotic region to nitroglycerin administration (arrow). 
ine and nicorandil.

One month later, the patient was admitted to hospital again for artificial urinary sphincter placement under spinal anesthesia, and had been on nifedipine until the morning of the day of surgery. Isosorbide dinitrate was continuously administered during surgery. Surgery was completed without intraoperative difficulties, and the patient was discharged without any complications.

\section{DISCUSSION}

CAS can induce angina and myocardial infarction in patients with no coronary artery disease [4]. Although the exact mechanism of CAS remains unclear, various intraoperative causes for CAS in susceptible patients have been reported, including increased activation of the sympathetic and parasympathetic nervous systems, functional anomalies of the vascular endothelium, imbalance of mediators involved in the vascular contraction-dilation process, drug use, hyperthyroidism, lipid metabolism disorder and metabolic factors, among many others $[2,5]$.

In our case, ST segment change occurred immediately after induction of spinal anesthesia and intravenous infusion of cefotetan; no drugs that directly affect the cardiovascular system had been used. We first considered anaphylactic shock due to cefotetan; however, it was ruled out because the laboratory tests for anaphylaxis were normal and no allergic reaction was observed when the drug was administered again. Therefore, we suspected the episode of ST segment change in the patient was due to CAS, and the cause of CAS was changes in the autonomic nervous system, especially sympathetic hyperactivity. Most cases of cardiac arrest after induction of regional anesthesia precede bradycardia or asystole due to sympathovagal response usually accompanied with high level of spinal blockade, leading to cardiac sympathetic block. However, our patient's block level and heart rate did not change after the onset of chest discomfort, so the probability of cardiac sympathetic fiber inhibition was minimal. Some cases of CAS occurred after administration of other drugs, including intravenous ephedrine, atropine or subcutaneous epinephrine [6-8]. Surgical stimulation with inadequate depth of anesthesia can be a trigger for CAS. But our patient had not received any adrenergic drugs and there was no surgical stimulation. Taniguchi et al. [9] reported car- diac sympathetic nerve activity was significantly increased in segments outside the anesthetized area during lumbar epidural anesthesia in cats. It is also reported that lumbar epidural anesthesia enhances arterial vascular tone and reactivity in sympathetically intact areas [10]. However, such reports can't reveal a direct relationship between sympathetic activity change after spinal anesthesia and CAS, thus we can only speculate that reflex sympathetic activity may involve CAS.

Although variant angina can be predicted when a patient has a history of chest pain accompanied by ST elevation, it is largely undetected during preoperative evaluations. Koshiba and Hoka [11] reviewed 115 patients who developed CAS during surgery, and reported that $85 \%$ did not have any signs of coronary artery disease. The patient described in the present report was also not suspected of having coronary artery disease based on preoperative tests. For early diagnosis of CAS, conscious patients must be carefully examined for symptoms, such as chest pain, and the cardiac rhythm must be continuously monitored. Patients with CAS may exhibit ST elevation or depression on ECG [12]. It is also useful to observe cardiac wall motion using transesophageal echocardiography. Observing reversible coronary constriction on coronary angiography is the definite diagnosis for CAS. However, coronary angiography could not be performed until 4 weeks after the event because we had difficulty in obtaining patient consent.

While CAS can be alleviated by nitroglycerin, without serious complications in most cases, symptoms similar to those of obstructive coronary ischemia can appear if CAS is not treated immediately and effectively and the patient can die from acute myocardial infarction and arrhythmia [13]. Therefore, early diagnosis, as well as rapid and appropriate treatment of CAS are important. In addition to anesthetic agents, anesthesiologists also administer antibiotics, blood products, and fluids rapidly at short intervals in the operating room. Anesthetic agents can cause hemodynamic changes through various mechanisms, and all drugs administered following anesthesia induction can cause allergic reactions. To differentiate between hemodynamic changes induced by anesthesia and allergic reactions caused by drug administration, there must be a time interval of sufficient length between anesthesia induction and drug administration. Patients also should pay attention to their symptoms, and immediately notify the surgery team or refer specifically to the anesthesi- 
ologist.

In conclusion, CAS can occur in patients with no history of coronary artery disease following induction of spinal anesthesia. Autonomic imbalance due to spinal anesthesia alone can cause CAS and cardiovascular collapse. It is important to promptly recognize ECG changes after anesthesia and take appropriate corrective action.

\section{REFERENCES}

1. Prinzmetal M, Kennamer R, Merliss R, Wada T, Bor N. Angina pectoris. I. A variant form of angina pectoris; preliminary report. Am J Med 1959; 27: 375-88.

2. Green MS, Gomes S. Intraoperative coronary artery vasospasm: a twist in the tale! Middle East J Anaesthesiol 2011; 21: 299-304.

3. Šoštarić S, Oremuš K. Sudden cardiorespiratory arrest following spinal anesthesia. Period Biol 2013; 115: 283-8.

4. Ornek E, Ornek D, Murat SN, Turfan M. Angiographic documentation of coronary artery spasm induced by anesthesia. J Anesth 2010; 24: 452-5.

5. Easley RB, Rosen RE, Lindeman KS. Coronary artery spasm during initiation of epidural anesthesia. Anesthesiology 2003; 99: 1015-7.

6. Hirabayashi Y, Saitoh K, Fukuda H, Mitsuhata H, Shimizu R. Cor- onary artery spasm after ephedrine in a patient with high spinal anesthesia. Anesthesiology 1996; 84: 221-4.

7. Wahl A, Eberli FR, Thomson DA, Luginbühl M. Coronary artery spasm and non-Q-wave myocardial infarction following intravenous ephedrine in two healthy women under spinal anaesthesia. Br J Anaesth 2002; 89: 519-23.

8. Lee JY, Hong SJ, Chon JY, Kwon SY. Cardiac arrest induced by submucosal injection of epinephrine in a patient with variant angina. Rhinology 2010; 48: 251-3.

9. Taniguchi M, Kasaba T, Takasaki M. Epidural anesthesia enhances sympathetic nerve activity in the unanesthetized segments in cats. Anesth Analg 1997; 84: 391-7.

10. Baron JF, Payen D, Coriat P, Edouard A, Viars P. Forearm vascular tone and reactivity during lumbar epidural anesthesia. Anesth Analg 1988; 67: 1065-70.

11. Koshiba K, Hoka S. Clinical characteristics of perioperative coronary spasm: reviews of 115 case reports in Japan. J Anesth 2001; 15: 93-9.

12. De Wolf AM, Kang YG. Coronary artery spasm and ST-segment depression. Anesthesiology 1985; 62: 368.

13. Sidi A, Dahleen L, Gaspardone A. Coronary vasospasm during anesthesia induction: awareness, recognition, possible mechanisms, anesthetic factors, and treatment. J Clin Anesth 2008; 20: 64-9. 